\title{
New-Onset Collagenous Colitis in a Patient With Psoriatic Arthritis: Can It Be Seculkinumab?
}

\author{
Adham E. Obeidat ${ }^{1}$, Traci T. Murakami ${ }^{2}$ \\ 1. Internal Medicine, University of Hawaii, Honolulu, USA 2. Gastroenterology and Hepatology, The Queen's Medical \\ Center West Oahu, Ewa Beach, USA
}

Corresponding author: Traci T. Murakami, tmurakami@queens.org

\begin{abstract}
Collagenous colitis is a chronic inflammatory condition and one type of a bigger entity, microscopic colitis. Collagenous colitis is associated with autoimmune diseases, such as psoriatic arthritis, suggesting an immune mechanism involved in the pathogenesis of the disease. New onset and flares of inflammatory bowel disease have been associated with the use of secukinumab, but no cases of microscopic colitis have been reported yet. We present a case of a 41 -year-old woman with psoriatic arthritis treated with secukinumab who developed chronic diarrhea and was found to have collagenous colitis.
\end{abstract}

Categories: Internal Medicine, Gastroenterology, Rheumatology

Keywords: collagenous colitis, colitis, diarrhea, secukinumab, psoriatic arthiritis, arthritis

\section{Introduction}

Collagenous colitis is a chronic inflammatory condition affecting the colon and it is a distinct type of inflammatory bowel disease (IBD). It usually presents with chronic watery diarrhea, abdominal pain, and weight loss $[1,2]$. Collagenous colitis has been associated with the use of some medications, most commonly non-steroidal anti-inflammatory drugs (NSAIDs) [2,3]. Furthermore, many cases of collagenous colitis have been associated with autoimmune diseases including rheumatoid arthritis, systemic lupus erythematosus (SLE), celiac disease, and, less frequently, psoriatic arthritis [2,4,5]. Recently, development of new cases and flares of ulcerative colitis (UC) and Crohn's disease (CD) have been associated with treatment with interleukin-17A (IL-17A) antagonist secukinumab, but to date, no cases of collagenous colitis have been reported [6-8]. Here, we report a case of a 41-year-old woman with psoriatic arthritis treated with secukinumab who was found to have collagenous colitis during an evaluation for chronic diarrhea.

Review began 06/13/2021 Review ended 06/20/2021 Published 07/03/2021

\section{๑) Copyright 2021}

Obeidat et al. This is an open access article distributed under the terms of the Creative Commons Attribution License CC-BY 4.0., which permits unrestricted use, distribution, and reproduction in any medium, provided the original author and source are credited.

\section{Case Presentation}

A 41-year-old woman with a past medical history of psoriatic arthritis, fibromyalgia, gastroesophageal reflux disease, and irritable bowel syndrome was referred to the gastrointestinal clinic for evaluation and management of chronic watery diarrhea. Three weeks before presentation, she had the onset of watery diarrhea, with no blood or mucus, which was recurring four times daily. Moreover, this was associated with waxing and waning abdominal pain, nausea, and unintentional weight loss of $3 \mathrm{~kg}$ in one week. The patient denied any vomiting, fever, chills, rigors, or urinary symptoms. A gluten-free and a BRAT (banana, rice, applesauce, and toast) diet did not help her symptoms. Patient's physical examination was unremarkable including her abdominal exam.

She had an upper endoscopy done three years earlier for persistent epigastric pain. Histopathology showed mild chronic gastritis with a normal esophagus and duodenum. She reported significant improvement in her epigastric pain until she developed a persistent watery diarrhea. Patient started taking venlafaxine 15 years prior to onset of her diarrhea. She also took NSAIDs for two years and sulfasalazine intermittently. She started taking secukinumab nine months prior to the onset of her diarrhea.

Blood and stool laboratory tests did not show any evidence of infection or malabsorption. Colonoscopy was done and it showed moderate mucosal congestion in transverse colon, ascending colon, and cecum, with non-bleeding internal hemorrhoids, but otherwise normal mucosa (Figure 1). Biopsies that were taken from multiple colonic sites and histopathological exam showed thickening of sub-epithelial basement membrane along with increased intraepithelial lymphocytes, which are consistent with the diagnosis of collagenous colitis (Figure 2). Immunological work-up including anti-nuclear antibodies, anti-centromere antibodies, anti-CCP antibodies, anti-snRNP antibodies, anti-Scl-70 antibodies, anti-SSA, and anti-SSB antibodies were all negative. The patient was started on budesonide with significant improvement in her symptoms. Budesonide was eventually tapered, and the patient was started on azathioprine as a steroid-sparing agent and immunomodulator to treat both collagenous colitis and psoriatic arthritis. 


\section{Cureus}



FIGURE 1: Colonoscopy image demonstrates moderate mucosal congestion. No polyps, nodules, or ulcerations.

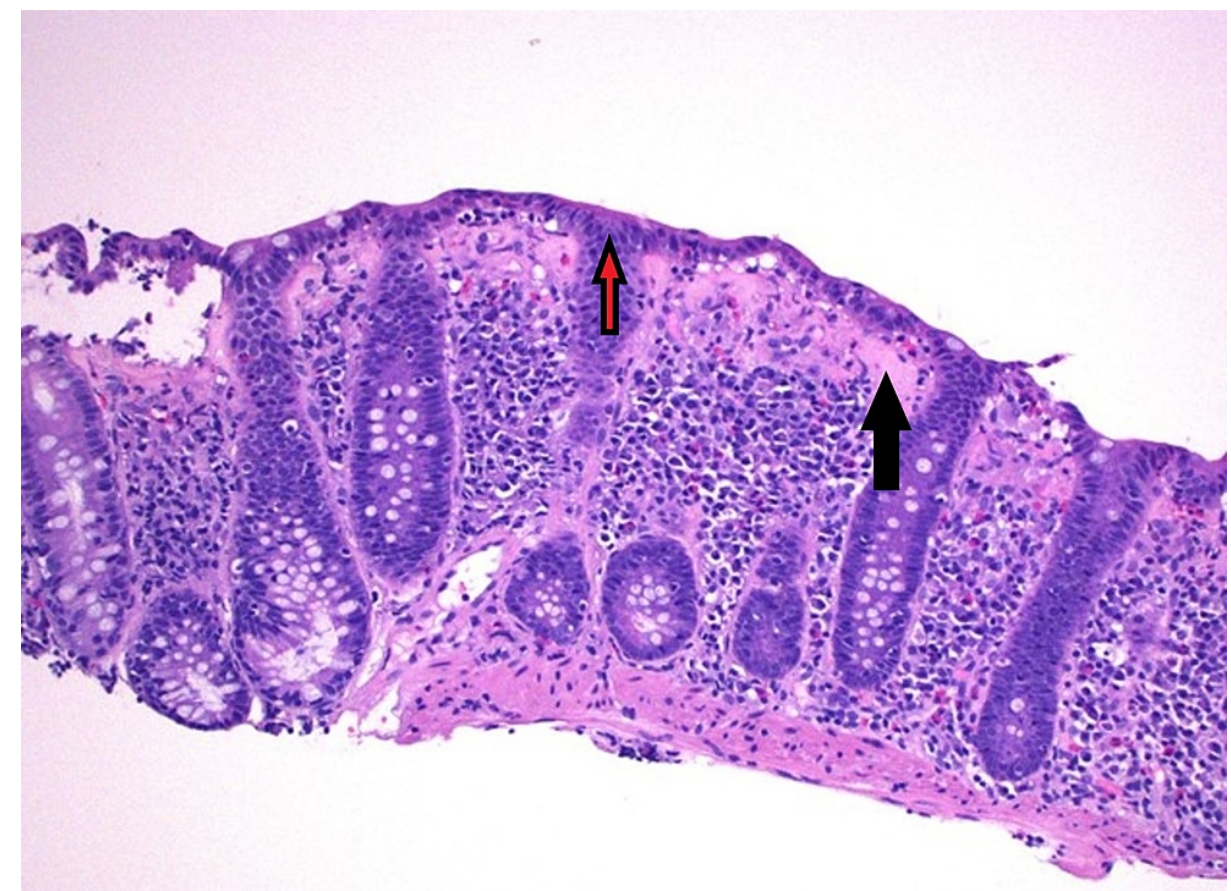

FIGURE 2: Microscopic image (H\&E stain, 100x power and 200x zoom) showing thickening of sub-epithelial basement membrane (black arrow) along with increased intra epithelial lymphocytes (red arrow).

\section{Discussion}

Microscopic colitis, which includes both collagenous colitis and lymphocytic colitis, is a chronic inflammatory condition of the colon, affecting females more than males and mostly people in their sixth or seventh decade of life $[1,2,9]$. The hallmark of collagenous colitis is chronic watery diarrhea. Collagenous colitis can present also with abdominal pain, weight loss, fecal incontinence, and bloody stool $[2,9]$. Laboratory and imaging studies are often normal, and the findings on colonoscopy are also normal. Diagnosis is made by performing biopsy and histopathological exam, which frequently shows diffuse 
thickening of the colonic sub-epithelial collagen layer, intraepithelial lymphocytosis, and infiltration of the lamina propria with inflammatory cells $[1,2,5,10]$.

Collagenous colitis is associated with multiple autoimmune diseases including rheumatoid arthritis, Hashimoto's thyroiditis, SLE, celiac disease, and spondyloarthropathies [2-4]. The first case of arthritis associated with collagenous colitis was described in 1983 by Erlendsson et al. [9]. Autoimmune disorders are associated with collagenous colitis in $20-60 \%$ of cases, and this association with autoimmune diseases raises the probability of an immune mechanism to be involved in the pathogenesis of the disease $[2,4,5]$.

Collagenous colitis is associated with some medications as well, most frequently NSAIDs. The mechanism is unclear, but it is suggested that NSAIDs inhibit prostaglandin synthesis, which increases colonic mucosal permeability, and this will lead to more intraluminal fluid, which in turn leads to increased risk of infection and inflammation. The length of NSAIDs use before the onset of symptoms of microscopic colitis can vary between few weeks to 15 years, and most people resolve directly after stopping the medication although some may need few months [3]. Our patient was taking NSAIDs intermittently for two years prior to onset of her diarrhea, but given the lack of improvement although she stopped using this medication, it is unlikely that it contributed to her symptoms. Other medications associated with the development of collagenous colitis include selective serotonin reuptake inhibitors, statins, and proton pump inhibitors, but the mechanism is unclear and most of the data came from descriptive and uncontrolled studies $[3,11,12]$.

The relationship between the use of secukinumab and the development of IBD is controversial. Some cases of UC and CD were reported in patients treated with secukinumab for various rheumatological diagnoses including ankylosing spondylitis, psoriatic arthritis, and plaque psoriasis. Duration of treatment varied between two and eight weeks prior to onset of symptoms $[6,8]$. On the other hand, two large studies were done to assess the efficacy of IL-17A antagonist in treating spondyloarthropathies, but they found no difference in the incidence of developing a new diagnosis or flares of IBD compared to placebo $[13,14]$. Moreover, two meta-analyses were conducted and they showed no increased risk of IBD with secukinumab in patients treated for ankylosing spondylitis, psoriasis, or psoriatic arthritis $[15,16]$.

The consideration of secukinumab as a potential trigger of IBD started in 2012 when Hueber et al. reported that secukinumab has not proven to be effective in treating active CD cases, and unexpectedly, there was an increased incidence of adverse events compared to placebo, which suggested a possible protective function of IL-17A in patients with CD [7]. Although causality has not been established yet, clinicians should be aware of the potential side effects of secukinumab. The mechanism of how secukinumab can lead to the development of IBD is unclear, but a protective function of IL-17A in maintaining intestinal barrier integrity has been described [17,18]. Furthermore, one study showed that neutralizing IL-17 enhanced the development of dextran sulfate sodium-induced colitis in mice by increasing tumor necrosis factor-alpha, interferon-gamma, and IL-6, which can promote the inflammatory response [19].

\section{Conclusions}

Our patient's symptoms started few months after starting secukinumab for the treatment of her psoriatic arthritis. Although a few cases of CD and UC associated with secukinumab use have been reported in the literature, this is the first case of collagenous colitis to be reported. Another possibility is that the patient had an undiagnosed collagenous colitis, and an acute flare has been triggered by the use of secukinumab. Although secukinumab is a safe and effective drug in treating psoriasis, psoriatic arthritis, and ankylosing spondylitis, it can be associated with rare but significant adverse events, which may lead to the development and trigger the flare of any of the spectrum of IBD from microscopic colitis to CD or UC, so physicians should be aware of these potential adverse events before starting the treatment.

\section{Additional Information \\ Disclosures}

Human subjects: Consent was obtained or waived by all participants in this study. Conflicts of interest: In compliance with the ICMJE uniform disclosure form, all authors declare the following: Payment/services info: All authors have declared that no financial support was received from any organization for the submitted work. Financial relationships: All authors have declared that they have no financial relationships at present or within the previous three years with any organizations that might have an interest in the submitted work. Other relationships: All authors have declared that there are no other relationships or activities that could appear to have influenced the submitted work.

\section{References}

1. Chande N, Driman DK, Reynolds RP: Collagenous colitis and lymphocytic colitis: patient characteristics and clinical presentation. Scand J Gastroenterol. 2005, 40:343-7. 10.1080/00365520510011623

2. Gopal P, McKenna BJ: The collagenous gastroenteritides: similarities and differences. Arch Pathol Lab Med. 2010, 134:1485-9. 10.5858/2010-0295-CR.1

3. Milman N, Kraag G: NSAID-induced collagenous colitis. J Rheumatol. 2010, 37:2432-3. 10.3899/jrheum.100573 
4. Azzouz D, Gargouri A, Hamdi W, et al.: Coexistence of psoriatic arthritis and collagenous colitis with inflammatory nervous system disease. Joint Bone Spine. 2008, 75:624-5. 10.1016/j.jbspin.2008.02.005

5. Ben Abdelghani K, Sahli H, Souabni L, et al.: Collagenous colitis and spondylarthropathy. Case Rep Med. 2012, 2012:620241. 10.1155/2012/620241

6. Ehrlich D, Jamaluddin N, Pisegna J, Padua D: A challenging case of severe ulcerative colitis following the initiation of secukinumab for ankylosing spondylitis. Case Rep Gastrointest Med. 2018, 2018:9679287. $10.1155 / 2018 / 9679287$

7. Hueber W, Sands BE, Lewitzky S, et al.: Secukinumab, a human anti-IL-17A monoclonal antibody, for moderate to severe Crohn's disease: unexpected results of a randomised, double-blind placebo-controlled trial. Gut. 2012, 61:1693-700. 10.1136/gutjnl-2011-301668

8. Rodríguez Moncada R, Vázquez Morón JM, Pallarés Manrique H: The onset of ulcerative colitis during treatment with secukinumab: can anti-IL-17A be a trigger for inflammatory bowel disease?. Rev Esp Enferm Dig. 2019, 111:720-1. 10.17235/reed.2019.5841/2018

9. Erlendsson J, Fenger C, Meinicke J: Arthritis and collagenous colitis. Report of a case with concomitant chronic polyarthritis and collagenous colitis. Scand J Rheumatol. 1983, 12:93-5. 10.3109/03009748309102892

10. Taccari E, Spada S, Giuliani A, Riccieri V, Sorgi ML, Pecorella I, Onetti Muda A: Co-occurrence of psoriatic arthritis with collagenous colitis: clinicopathologic findings of a case. Clin Rheumatol. 2002, 21:335-8. 10.1007/s100670200088

11. Beaugerie L, Pardi DS: Review article: drug-induced microscopic colitis - proposal for a scoring system and review of the literature. Aliment Pharmacol Ther. 2005, 22:277-84. 10.1111/j.1365-2036.2005.02561.x

12. Verhaegh BP, de Vries F, Masclee AA, et al.: High risk of drug-induced microscopic colitis with concomitant use of NSAIDs and proton pump inhibitors. Aliment Pharmacol Ther. 2016, 43:1004-13. 10.1111/apt.13583

13. Baraliakos X, Kivitz AJ, Deodhar AA, et al.: Long-term effects of interleukin-17A inhibition with secukinumab in active ankylosing spondylitis: 3-year efficacy and safety results from an extension of the Phase 3 MEASURE 1 trial. Clin Exp Rheumatol. 2018, 36:50-5.

14. Baeten D, Sieper J, Braun J, et al.: Secukinumab, an interleukin-17A inhibitor, in ankylosing spondylitis . N Engl J Med. 2015, 373:2534-48. 10.1056/NEJMoa1505066

15. Schreiber S, Colombel JF, Feagan BG, et al.: Incidence rates of inflammatory bowel disease in patients with psoriasis, psoriatic arthritis and ankylosing spondylitis treated with secukinumab: a retrospective analysis of pooled data from 21 clinical trials. Ann Rheum Dis. 2019, 78:473-9. 10.1136/annrheumdis-2018-214273

16. Schreiber S, Sands B, Deodhar A, et al.: OP0113 no increased incidence of inflammatory bowel disease among secukinumab-treated patients with moderate to severe psoriasis, psoriatic arthritis, or ankylosing spondylitis: data from 14 phase 2 and phase 3 clinical studies. Ann Rheum Dis. 2016, 75:97.2-98. 10.1136/annrheumdis-2016-eular.3684

17. Whibley N, Gaffen SL: Gut-busters: IL-17 ain't afraid of no IL-23 . Immunity. 2015, 43:620-2. 10.1016/j.immuni.2015.10.001

18. Ruiz de Morales JM, Puig L, Daudén E, et al.: Critical role of interleukin (IL)-17 in inflammatory and immune disorders: An updated review of the evidence focusing in controversies. Autoimmun Rev. 2020, 19:102429. 10.1016/j.autrev.2019.102429

19. Ogawa A, Andoh A, Araki Y, Bamba T, Fujiyama Y: Neutralization of interleukin-17 aggravates dextran sulfate sodium-induced colitis in mice. Clin Immunol. 2004, 110:55-62. 10.1016/j.clim.2003.09.013 